\title{
$N$-Acetylglutaminoyl-S-farnesyl-L-cysteine (SIG-1191): an anti- inflammatory molecule that increases the expression of the aquaglyceroporin, aquaporin-3, in human keratinocytes
}

\author{
José R. Fernández ${ }^{1} \cdot$ Corey Webb $^{1} \cdot$ Karl Rouzard $^{1} \cdot$ Michael Voronkov $^{1}$. \\ Kristen L. Huber ${ }^{1} \cdot$ Jeffry B. Stock $^{1,2} \cdot$ Maxwell Stock $^{1} \cdot$ Joel S. Gordon $^{1}$ • \\ Eduardo Perez ${ }^{1}$
}

Received: 14 June 2016/Revised: 5 December 2016/Accepted: 8 December 2016/Published online: 17 December 2016

(C) The Author(s) 2016. This article is published with open access at Springerlink.com

\begin{abstract}
Isoprenylcysteine (IPC) small molecules were discovered as signal transduction modulating compounds $\sim 25$ years ago. More recently, IPC molecules have demonstrated antioxidant and anti-inflammatory properties in a variety of dermal cells as well as antimicrobial activity, representing a novel class of compounds to ameliorate skin conditions and disease. Here, we demonstrate a new IPC compound, $\quad N$-acetylglutaminoyl- $S$-farnesyl-L-cysteine (SIG-1191), which inhibits UVB-induced inflammation blocking pro-inflammatory cytokine interleukin-6 (IL-6) and tumor necrosis factor alpha (TNF- $\alpha$ ) production. To investigate further the previously reported hydrating potential of IPC compounds, SIG-1191 was tested for its ability to modulate aquaporin expression. Specifically, aquaporin 3 (AQP3) the most abundant aquaporin found in skin has been reported to play a key role in skin hydration, elasticity and barrier repair. Results show here for the first time that SIG-1191 increases AQP3 expression in both cultured normal human epidermal keratinocytes as well as when applied topically in a three-dimensional (3D) reconstructed human skin equivalent. Additionally, SIG1191 dose dependently increased AQP3 protein levels, as determined by specific antibody staining, in the epidermis of the 3D skin equivalents. To begin to elucidate which signaling pathways SIG-1191 may be modulating to increase AQP3 levels, we used several pharmacological pathway inhibitors and determined that $\mathrm{AQP} 3$ expression is
\end{abstract}

Eduardo Perez

eperez@signumbio.com

1 Signum Dermalogix, 133 Wall Street, Princeton, NJ 08540, USA

2 Department of Molecular Biology, Princeton University, Princeton, NJ, USA mediated by the Mitogen-activated protein kinase/Extracellular signal-regulated kinase kinase (MEK) pathway. Altogether, these data suggest SIG-1191 represents a new IPC derivative with anti-inflammatory activity that may also promote increased skin hydration based on its ability to increase AQP3 levels.

Keywords Aquaglyceroporin-3 · Skin hydration · Isoprenylcysteine $\cdot$ Inflammation

\section{Introduction}

The addition of farnesyl (15-carbon side chain) or geranylgeranyl (20-carbon side chain) isoprenoids is essential to the membrane targeting of heterotrimeric and small $\mathrm{G}$ proteins that mediate receptor signaling in eukaryotic cells. This post-translational modification occurs within a CAAX-tail motif, where the isoprenoid is attached to a cysteine residue via a thioether bond [48]. Isoprenylcysteine (IPC) analogs contain the same 15- or 20-carbon side chain mimicking the $\mathrm{C}$ terminus of processed CAAX proteins [52]. IPC analogs upon cellular uptake affect signaling activities by several mechanisms. First, through insertion into the membrane, they compete with isoprenoid groups for prenyl-binding sites on membrane-associated proteins [11, 30, 34, 43]. In addition, IPC analogs modulate signal transduction by inhibiting heterotrimeric $\mathrm{G}$ protein formation $[12,17]$ and by binding and activating peroxisome proliferator-activated receptor gamma (PPAR $\gamma$ ) [6].

IPC analogs have been initially identified as a novel class of topical anti-inflammatory compounds [15, 16, 19]. In vitro studies have shown IPC compounds to be effective down modulators of inflammatory responses in platelets, macrophages and neutrophils [25, 30, 41, 50]. IPC 
derivatives were shown to inhibit pro-inflammatory TNF- $\alpha$ stimulation of vascular cell adhesion molecule-1 (VCAM1) by modulating small $G$ protein Rac1 activity [3, 39] and suppressing purinergic receptor (a $G$ protein-coupled receptor-GPCR)-mediated IL-8, monocyte chemotactic protein-1 (CCL2) and growth-regulated oncogene $\alpha$ (CXCL1) production [1]. Recent studies demonstrate IPC analogs also downregulate non-G protein-mediated inflammation in human epidermal keratinocytes, dermal fibroblasts and peripheral blood mononuclear cells by abrogating toll-like receptor 2 (TLR2), toll-like receptor 4 (TLR4) and $\mathrm{T}$ cell receptor (TCR) signaling [14]. Furthermore, IPC analogs have also been shown to inhibit ultraviolet A (UVA), ultraviolet B (UVB), phorbol 12-myristate 13-acetate (TPA) and bacteria-induced proinflammatory cytokine release $[14,15,19]$ in skin cells, highlighting the effectiveness of this class of compounds in blocking cutaneous inflammation. Interestingly, we have also shown that in a human use study, IPCs have the potential to enhance skin hydration [16].

Aquaporins (AQPs) are a family of highly conserved transmembrane proteins that act primarily as water-selective pores, facilitating the transport of water across cell membranes [10]. Thirteen mammalian AQPs have been identified over the last 23 years with differing functions dependent on localization [23]. To date, AQP3, AQP9 and AQP10, called aqua-glycoporins because they transport water, glycerol and possibly other small solutes, have been found in skin [28, 42]. AQP3, the most abundant AQP found in skin, is located in the basal, suprabasal and stratum corneum layers [2, 18, 28, 33, 51] of the epidermis at the cell periphery [47]. The critical role(s) AQP3 plays in skin hydration were elucidated using AQP3 knockout mice. These studies demonstrated mice lacking AQP3 had reduced stratum corneum hydration and elasticity, exhibiting delayed barrier recovery and wound healing [21]. In addition, altered AQP3 expression and production have also been linked to several skin diseases such as eczema [9, 38], psoriasis, non-melanoma skin cancers [23, 31, 44, 52] and vitiligo [29]. Recently, AQP3 has also been shown to play a protective role in UV-induced apoptosis in fibroblasts [54] and has also been suggested to be a participating factor in skin aging [45]. Thus, AQP3 is a key player in the skin and identifying compounds that modulate its activity could yield new therapeutic actives for drug development and skin care.

Here, we report that IPC analog $N$-acetylglutaminoyl- $S$ farnesyl-L-cysteine (SIG-1191) (Fig. 1a) in which a glutamine is thioacetylated to the cysteine residue possesses anti-inflammatory properties by inhibiting UV-induced pro-inflammatory mediators in human keratinocytes, similar to previous IPC compounds [15, 16]. Furthermore, we demonstrate for the first time that SIG-1191 upregulates
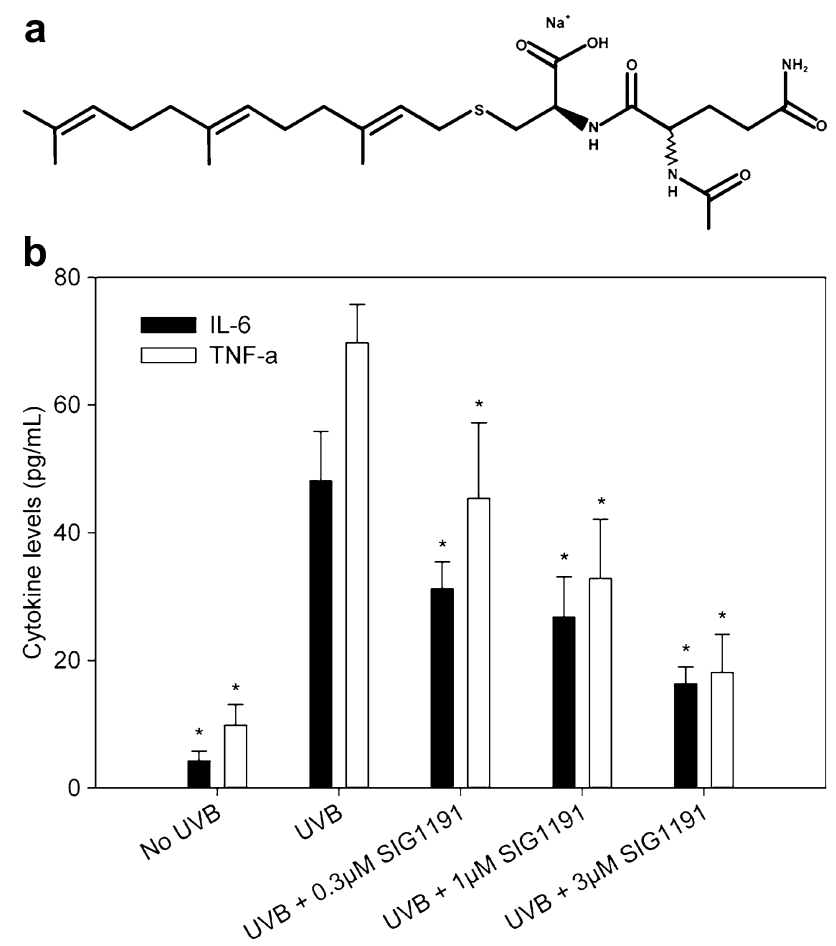

Fig. 1 SIG-1191 has anti-inflammatory activity blocking UVB proinflammatory cytokine production. a Chemical structure of monosodium salt of $N$-acetylglutaminoyl- $S$-farnesyl-L-cysteine (SIG-1191) b Cells were incubated with SIG-1191 for $6 \mathrm{~h}$, washed and irradiated in PBS with $25 \mathrm{~mJ} / \mathrm{cm}^{2}$ UVB and later incubated without SIG-1191 in supplement-depleted media for $24 \mathrm{~h}$. Pro-inflammatory cytokines (IL6 and TNF- $\alpha$ ) were measured from media supernatants by ELISA. The data represent the mean \pm SEM of cumulative from three independent experiments. $* p<0.05$ indicates a statistically significant difference compared to UVB-only irradiated cells

AQP3 gene expression in monolayer keratinocytes and both gene expression and protein production in reconstructed three-dimensional human skin cultured at the airliquid interface. Using specific pathway inhibitors, induction of AQP3 expression is demonstrated to be mediated by MAPK kinase (MEK)-dependent pathways. Altogether, these results suggest SIG-1191 may be effective in boosting skin hydration, elasticity and barrier recovery through its ability to increase AQP3 levels in addition to reducing inflammation.

\section{Materials and methods}

\section{Reagents}

SIG-1191 ( $N$-acetylglutaminoyl-S-farnesyl-L-cysteine) was synthesized according to the methods described in US patent application US 12/616,781. The monosodium salt of SIG-1191 was used for all assay treatments (Fig. 1a). All chemicals were analyzed by LC/MS (Agilent 1100), ${ }^{1} \mathrm{H}$ 
and ${ }^{13} \mathrm{C}$ NMR (500 and $125 \mathrm{MHz}$, Bruker) for structural identity and confirmed to be $>93 \%$ pure by analytical HPLC (Agilent 1200; Santa Clara, CA).

\section{Monolayer NHEK cell and 3D reconstructed human skin tissue culture}

Normal human epidermal keratinocytes (NHEKs) from neonatal donors were obtained from ThermoFisher (Carlsbad, CA). Cells were cultured until the second passage and seeded on six-well plates for $24 \mathrm{~h}$ before treatments with EpiLife $^{\circledR}$ media supplemented with keratinocyte growth supplement and $60 \mu \mathrm{M}$ calcium (ThermoFisher; Carlsbad, CA).

The EpiDerm-FT ${ }^{\mathrm{TM}}$ skin cultures, reconstructed from cells of human origin, were purchased as preserved culture inserts from MatTek Corp. (Ashland, MA). They were cultured and acclimated at the air-liquid interface at $37{ }^{\circ} \mathrm{C}$ and $5 \% \mathrm{CO}_{2}$ for $24 \mathrm{~h}$ in six-well plates. SIG-1191 formulated in water was single administered by topical application $(25 \mu \mathrm{L})$ and incubated for $24 \mathrm{~h}$.

\section{Gene expression}

NHEK cell monolayers and EpiDerm-FT ${ }^{\mathrm{TM}}$ tissues were treated for $24 \mathrm{~h}$. Harvested cells and biopsy punches $(8 \mathrm{~mm})$ were obtained from EpiDerm-FT ${ }^{\mathrm{TM}}$ cultures after treatments and homogenized using a Dounce homogenizer (1 mL capacity). Total RNA was extracted using the RNAqueous kit $\left(\right.$ Ambion $^{\circledR}$ ) and cDNA was obtained using the High-Capacity RNA-to-cDNA kit (Applied Biosystems). Quantitative PCR (qPCR) was performed using the TaqMan ${ }^{\circledR}$ Fast Advanced Master Mix (Applied Biosystems) and specific TaqMan ${ }^{\circledR}$-probes human gene primers for AQP3, AQP9 and GAPDH to calculate the relative gene fold expression change per treatment. Gene expression analysis was performed using the comparative $\mathrm{Ct}$ method (2-[delta][delta] $\mathrm{Ct}$ ) approach by comparing the $\mathrm{Ct}$ values of the treated samples with the untreated samples and normalized to GAPDH gene expression as endogenous housekeeping gene.

\section{Histology}

After 24-h treatments, EpiDerm-FT ${ }^{\mathrm{TM}}$ cultures were fixed in $10 \%$ formalin and later preserved by paraffin embedding. Sections $(5 \mu \mathrm{m})$ were stained with hematoxylin and eosin (H\&E) for evaluation of tissue morphology. Immunohistochemistry (IHC) was performed using anti-aquaporin-3 (AbCam; Cambridge, UK) and anti-keratin-10 (EMD Millipore; Billerica, MA). Secondary antibodies used were anti-rabbit-Alexa Fluor-488 and anti-mouse-Alexa Fluor594 (Jackson Immuno Research Labs; West Grove, PA).
Antibody specificity was confirmed by staining tissues with secondary antibodies only. Stained sections were viewed using an Olympus BX-41 microscope (Center Valley, PA) equipped with CCD camera (Hamamatsu Photonics K.K.; Shizuoka Pref., Japan) and using MetaMorph ${ }^{\circledR}$ software (Molecular Devices, Sunnyvale, CA).

\section{UVB radiation}

Monolayer NHEKs were treated with SIG-1191 monosodium salt $(0,0.3,1,3 \mu \mathrm{M})$ for $6 \mathrm{~h}$ at $37^{\circ} \mathrm{C}$ and $5 \% \mathrm{CO}_{2}$ in depleted media (without growth factors). Later, treatment media was removed, cells were washed $3 \times$ with PBS and irradiated in PBS (phenol red-free) with $25 \mathrm{~mJ} / \mathrm{cm}^{2}$ UVB using an external research irradiator (Daavlin Co, Bryan, $\mathrm{OH}$ ) equipped with broadband UVB lamps $(305 \pm 12 \mathrm{~nm})$. After irradiation, media was replaced with depleted media without SIG-1191 and incubated for $24 \mathrm{~h}$ at $37{ }^{\circ} \mathrm{C}$ and $5 \% \mathrm{CO}_{2}$. After incubation, media supernatants were obtained and human IL- 6 and TNF $\alpha$ were measured by standard sandwich ELISA following the recommended manufacturer's protocol (BD Biosciences; San Jose, CA).

\section{Statistical analysis}

Statistical significance was determined by one-way ANOVA followed by Bonferroni multiple comparisons test using $p$ values less than 0.05 as a significant difference.

\section{Results}

\section{SIG-1191 inhibits UVB-induced pro-inflammatory cytokine production}

UVB-induced photoaging is mediated through production of inflammatory cytokines and MMPs induced through the activation of the MAP kinase and NFKB pathways [32]. The resulting induction of interleukin-6 (IL-6) and tumor necrosis factor alpha (TNF- $\alpha$ ) also contributes to skin dryness [40]. We tested SIG-1191 to determine if it possessed similar characteristics to previously tested IPC analogs [15], specifically by decreasing UVB-induced proinflammatory cytokine release from cultured primary keratinocytes (NHEKs). Our results show SIG-1191 dose dependently inhibits IL-6 and TNF- $\alpha$ release with an $\mathrm{IC}_{50}=1 \mu \mathrm{M}$ (Fig. 1b).

\section{SIG-1191 increases AQP3 gene expression in epidermal cells}

UVB exposure damages skin water barrier function as determined by increased transepidermal water loss 
(TEWL) [24] and potentially downregulation of AQPs [26]. Since IPC compounds have been shown to improve hydration and skin firmness in a clinical study [16], we sought to determine if SIG-1191 could modulate the expression of human skin epidermal aquaporins (AQP3, AQP9). To investigate aquaporin gene expression activity, NHEK cells were treated with the indicated concentrations of SIG-1191 in media for $24 \mathrm{~h}$. After incubation, cells were collected and gene expression was assessed by quantitative PCR (qPCR). After $24 \mathrm{~h}$, SIG-1191 at $\geq 3 \mu \mathrm{M}$ significantly increased AQP3 gene expression +216-609\% (Fig. 2a). Conversely, SIG-1191 only at the highest concentrations tested $(\geq 10 \mu \mathrm{M})$ had a slight, but significant decrease on AQP9 expression (30\% reduction), an understandable
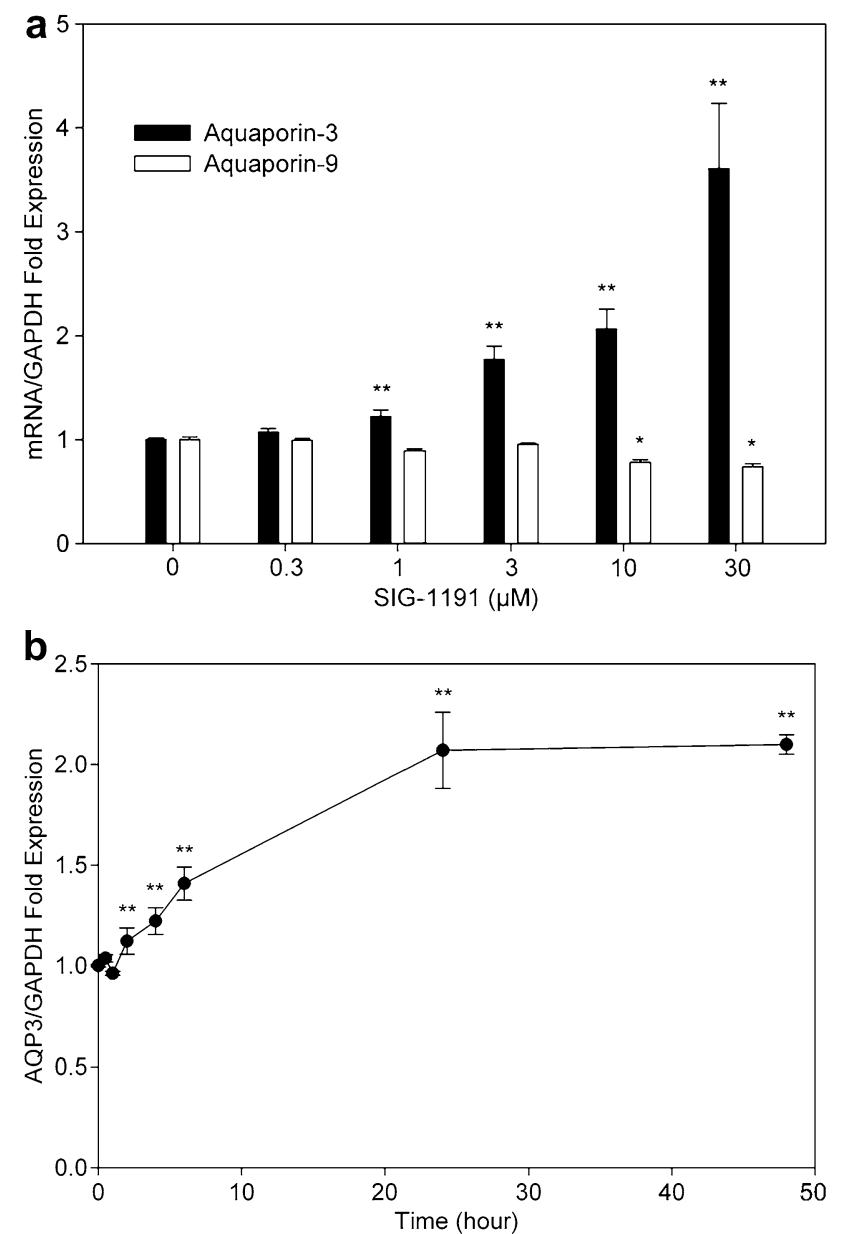

Fig. 2 SIG-1191 increases AQP3 gene expression in a dose- and time-dependent manner. a NHEKs were treated with the indicated concentrations of SIG-1191 for $24 \mathrm{~h}$. b Cells were treated with $10 \mu \mathrm{M}$ SIG-1191 $(2,4,6,8,24,48 \mathrm{~h})$ and harvested for gene expression analysis. The level of gene expression of aquaporins (AQP3, AQP9) was quantitated by qPCR normalizing to level of GAPDH the control housekeeping gene. The data represent the mean \pm SEM of cumulative from three independent experiments. ${ }^{*} p<0.05$; $* * p \leq 0.01$ indicates a statistically significant difference compared to untreated cells result given that AQP9 expression in keratinocytes has been reported to be regulated in a different manner than that of AQP3 [49]. SIG-1191 significantly increased AQP3 gene expression after only $2 \mathrm{~h}$ of exposure and reaching maximum levels at $24-48 \mathrm{~h}$ (Fig. 2b). After 48-h treatments, the increased expression of AQP3 remained stable.

\section{Increase of AQP3 expression is mediated by the MEK pathway}

The mRNA levels of AQP3 in epidermal keratinocytes have been shown to be dependent on several signaling pathways including those that involve phosphatidylinositol 3-kinase and MAPK kinases [4, 9, 20, 27]. In addition, AQP3 has been shown to associate with phospholipase D2 (PLD2) to promote keratinocyte differentiation pathway [7]. Therefore, we used pharmacological agents that inhibit these specific signaling pathways to investigate which signal(s) is (are) used by SIG-1191 to modulate AQP3 expression. NHEKs were treated with phospholipase D2 (CAY10594), phosphatidylinositol 3-kinase (LY294002) and MAPK kinases (U0126) inhibitors and co-treated with SIG-1191 $(10 \mu \mathrm{M})$ in media for $24 \mathrm{~h}$. After incubation, cells were collected and AQP3 gene expression was assessed by qPCR. Results show that U0126 alone significantly reduced AQP3 mRNA levels compared to untreated cells. In addition, U0126 inhibits the SIG-1191-induced increase in AQP3 expression (Fig. 3), consistent with the involvement of MAPK kinase (MEK)-dependent signaling pathway in SIG-1191-induced AQP3 expression in NHEKs.

\section{SIG-1191 increases AQP3 gene and protein expression in a 3D human skin model}

SIG-1191-induced AQP3 expression was validated using the full-thickness EpiDerm ${ }^{\mathrm{TM}}$ reconstructed human skin model (MatTek, Corp.) cultured at the air-liquid interface. Tissues were topically treated with SIG-1191 for $24 \mathrm{~h}$ and AQP3 mRNA accumulation was assessed by qPCR. SIG1191 at $0.25-0.5 \% \mathrm{w} / \mathrm{v}$ in a dose-dependent manner significantly increased AQP3 gene expression +322 - and 456-fold, respectively, after $24 \mathrm{~h}$ (Fig. 4a). Topical treatment of the reconstructed skin cultures showed no alteration of the morphology as revealed by H\&E staining, nor was there any effect on the level of staining of suprabasal keratin-10 (K10) (Fig. 4b). AQP3 antibody staining localized to basal layer and partially to the lowest suprabasal layer, predominantly restricted to the cell periphery in untreated and vehicle-exposed cultures (Fig. 4b). Treatment with SIG-1191 increased in an apparent dose-dependent manner the intensity and distribution of AQP3 staining. For example, after treatment with SIG-1191 at 


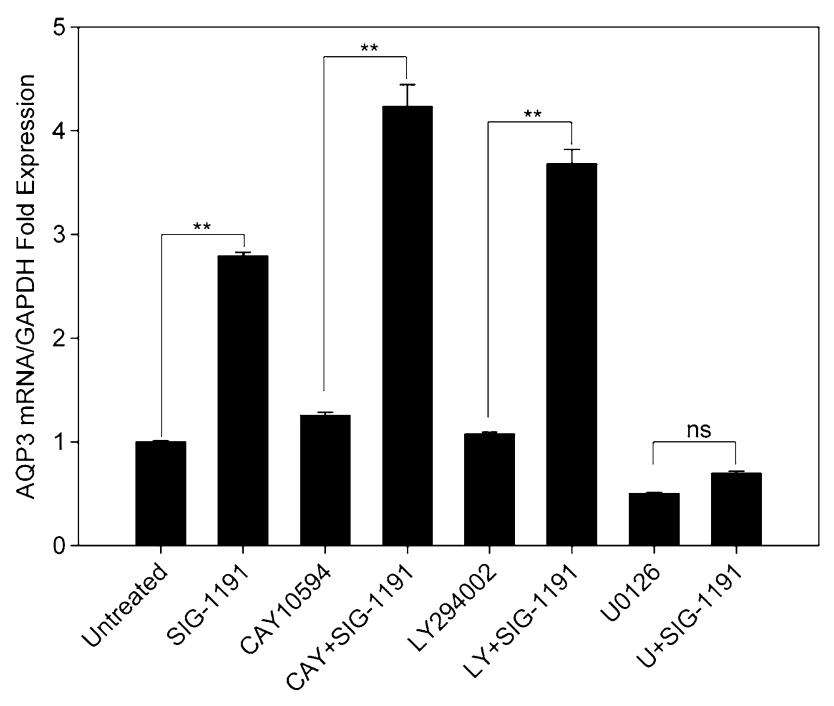

Fig. 3 AQP3 gene expression is activated by the MEK pathway. NHEKs were treated with $10 \mu \mathrm{M}$ SIG-1191 with and without the indicated pathway inhibitors (1 $\mu \mathrm{M}$ CAY10594 (CAY) a phospholipase D2 inhibitor; $10 \mu \mathrm{M}$ LY294002 (LY) a phosphatidylinositol 3-kinase inhibitor; $10 \mu \mathrm{M}$ U0126 (U) a MAPK/ERK kinase inhibitor) for $24 \mathrm{~h}$ and harvested for AQP3 mRNA levels. Gene expression of AQP3 was analyzed by qPCR normalized to GAPDH, a control housekeeping gene. The data represent the mean \pm SEM of cumulative from three independent experiments. $* p<0.05$; $* p \leq 0.01$ indicates a statistically significant difference compared to untreated cells ( $n s$ not significant)

$0.25 \%$, AQP3 protein expression is visualized in the midsuprabasal layers, while SIG-1191 applied at $0.5 \%$ increased overall intensity of staining, with AQP3 now seen throughout the cell cytoplasm and cell periphery staining detected in the suprabasal layers. This observation is emphasized by overlaying $\mathrm{K} 10$ and AQP3 antibody staining (Fig. 4b). Thus, when applied topically, SIG-1191 induces an increase in AQP3 mRNA expression and protein production.

\section{Discussion}

In this study, we set to characterize the properties of $\mathrm{N}$ acetylglutaminoyl-S-farnesyl-L-cysteine (SIG-1191), a novel IPC analog. The archetype of the farnesylated class of IPC molecules, $N$-acetyl- $S$-farnesyl-L-cysteine (AFC) was discovered $\sim 25$ years ago [50] and was subsequently shown to block chemically induced inflammation when applied topically in vivo [19]. Moreover, a different IPC farnesyl derivative called SIG-990 has also demonstrated anti-inflammatory activity and is being developed as a potential therapeutic for rosacea. Another farnesyl IPC, $S$ farnesylthiosalicylic acid has been shown to target inflammation in animal models of contact dermatitis and allergic inflammation [36, 37]. Building upon these results, we found that SIG-1191, similar to its IPC predecessors, effectively reduces inflammation as shown by the inhibition of UVB-induced IL- 6 and TNF- $\alpha$ (Fig. 1b) production with greater potency than AFC in NHEKs (unpublished results). This suggests that the chemical modification of adding a glutamine residue to the IPC chemical backbone confers additional anti-inflammatory activity.

Aquaporins (AQPs) are pore-like passive channel transporters of water or glycerol that are widely expressed. AQP3 and AQP9 are expressed by human skin and have been shown to contribute to stratum corneum (SC) hydration and glycerol transport [13]. AQP3-null mice showed high-frequency skin conductance and ${ }^{3} \mathrm{H}_{2} \mathrm{O}$ partitioning was reduced compared to wild-type animals, suggesting the critical role of AQP3 in SC hydration [21]. AQP3 is mostly expressed at the basal layer of the epidermis [46] and its expression is downregulated by UVB irradiation $[26,46]$. It is present in both viable and stratum corneum layers of the epidermis [28], thus suggesting the functional role in water transport. Interestingly, the water loss prevention of the stratum corneum is linked to the specific expression of AQP3 between the dermis and basal layers of the epidermis, thus operating as a "water-clamp" to prevent water loss gradient from the dermis to the stratum corneum [47]. Since we have previously demonstrated that IPCs enhance skin hydration in human use studies [16] we utilized SIG1191 to elucidate the possible molecular basis for this enhancement of skin hydration. To this end, we selected AQP3 and AQP9 as initial targets. Our results show primary keratinocytes exposed to $\geq 3 \mu \mathrm{M}$ SIG-1191 after $24 \mathrm{~h}$ induce an increase in AQP3 gene expression through activation of a specific signaling pathway with a minimal decrease on AQP9 expression (Fig. 2). When topically applied on human reconstructed skin $\left(\right.$ EpiDerm-FT ${ }^{\mathrm{TM}}$ model), SIG-1191 induced AQP3 mRNA overexpression (Fig. 4a) and via immunohistological analysis, AQP3 was visibly overexpressed $24 \mathrm{~h}$ after treatment of treatment with SIG-1191 (Fig. 4b).

AQP3 expression is upregulated by both nuclear and membrane receptor activation [53]. Another commonly used skin care compound, retinoic acid has been shown to increase AQP3 and also slightly decrease AQP9 gene and protein expression via the nuclear retinoic acid receptor subtype gamma $(\operatorname{RAR} \gamma)[5,49]$. The involvement of this receptor in any potential SIG-1191 AQP3-mediated enhancement of skin hydration is unlikely since retinoids are associated with skin drying. The upregulation of AQP3 is more likely part of the barrier repair response secondary to the drying effects of retinoic acid. Other studies demonstrate the nuclear PPAR $\gamma$ receptor stimulates AQP3 expression in keratinocytes [27]. Previous studies showed IPCs were ligands for PPAR $\gamma$ [6]. However, reporter assays with SIG-1191 revealed it was not a PPAR $\gamma$ activator (unpublished results). 


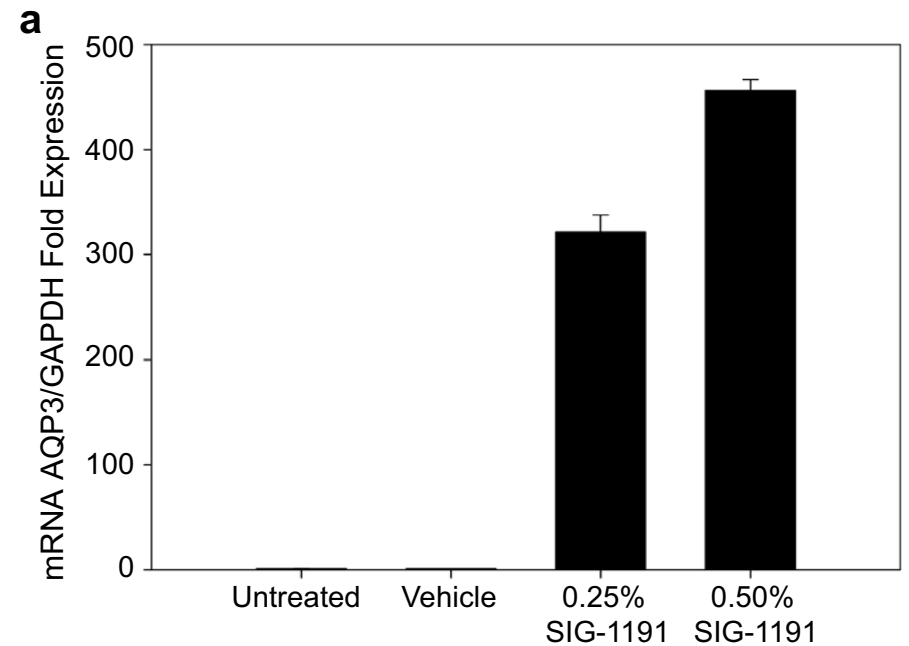

b

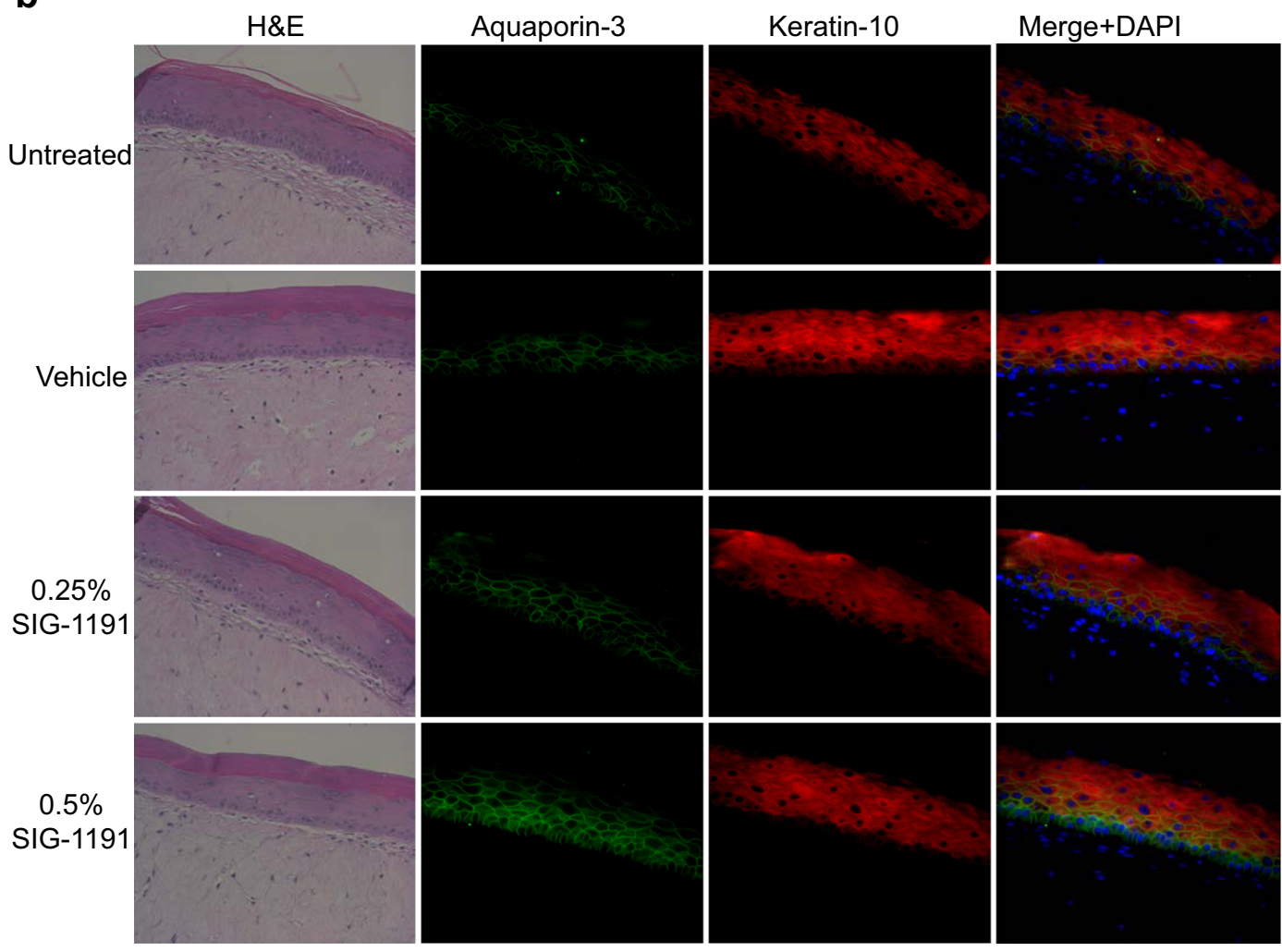

Fig. 4 SIG-1191 increases AQP3 gene expression and protein levels in Reconstructed Human Epidermis (RHE). EpiDerm-FT ${ }^{\mathrm{TM}}$ airliquid interface cultures were topically treated with $0.25-0.5 \%(\mathrm{w} / \mathrm{v})$ of SIG-1191 for $24 \mathrm{~h}$. a AQP3 gene expression was analyzed by qPCR normalized to GAPDH a control housekeeping gene. The data represent the mean \pm SEM of a representative experiment. b Haematoxylin and eosin (H\&E) staining and immunohistochemistry (IHC) of EpiDerm-FT ${ }^{\mathrm{TM}}$ tissues. Immunohistochemistry was performed

Since, AQP3 gene expression is regulated by the activation of transcription factors by different membrane receptor-activated signaling pathways $[4,7,9,20]$, using specific inhibitors to each of the known pathways, we found SIG-1191 controls AQP3 gene expression through with anti-aquaporin-3 (green), anti-keratin-10 (red), anti-rabbit Alexa-488, anti-mouseAlexa-594 antibodies. Sections were counterstained with 2-(4-amidinophenyl)-1H-indole-6-carboxamidine (DAPI). Merged micrograph shows overlaying K10 and AQP3 antibodies with DAPI staining. No background fluorescence was observed in the absence of the primary antibody (not shown). Original magnification: $\times 400$

the MAPK/ERK kinase pathway (Fig. 3). Activation of this pathway has been demonstrated to be critical for skin hydration and re-epithelialization during wound healing [9, 22, 35]. IPC derivatives shown to modulate either GPCR or toll-like receptor (TLR)-activated pathways are 
not known to regulate AQP3 expression or to generally signal through the MEK pathway. The small G protein Ras, which has been previously shown to be modulated by IPCs, signals via the MAPK kinase pathway [41]. Thus, the possibility of this pathway remains open to be modulated by IPC compounds, until it is determined which Ras-dependent pathway plays a role in regulating AQP3 expression. It should be noted that IPC analogs have only been shown to downregulate Ras signaling and increased expression of AQP3 would require that SIG-1191 upregulates Ras signaling. Specifically, the EGF receptor, which activates the MEK pathway through Ras, is known to upregulate AQP3 expression in keratinocytes [9]. Therefore, it is possible that the EGF receptor is also IPC sensitive or that SIG-1191 modulates GPCR and/or TLRactivated pathways that impinge on MEK signaling.

\section{Compliance with ethical standards}

Conflict of interest JSG is a paid consultant for Signum Dermalogix, while JSF, CW, KR, MV, MS, KLH and EP are employees. JBS serves on the board of directors. All authors have stock and/or stock options in the company.

Open Access This article is distributed under the terms of the Creative Commons Attribution 4.0 International License (http://crea tivecommons.org/licenses/by/4.0/), which permits unrestricted use, distribution, and reproduction in any medium, provided you give appropriate credit to the original author(s) and the source, provide a link to the Creative Commons license, and indicate if changes were made.

\section{References}

1. Adhami K, Lee J et al (2012) $N$-acetyl-S-farnesyl-L-cysteine suppresses chemokine production by human dermal microvascular endothelial cells. Exp Dermatol 21:700-705

2. Agren J, Zelenin S, Svensson LB, Nejsum LN, Nielsen S, Aperia A, Sedin G (2010) Antenatal corticosteroids and postnatal fluid restriction produce differential effects on $\mathrm{AQP} 3$ expression, water handling, and barrier function in perinatal rat epidermis. Dermatol Res Pract. doi:10.1155/2010/789729

3. Ahmad M, Zhang Y et al (2002) Role of isoprenylcysteine carboxyl methyltransferase in tumor necrosis factor-alpha stimulation of expression of vascular cell adhesion molecule-1 in endothelial cells. Arterioscler Thromb Vasc Biol 22:759-764

4. Bell CE, Lariviere NM et al (2009) Mitogen-activated protein kinase (MAPK) pathways mediate embryonic responses to culture medium osmolarity by regulating Aquaporin 3 and 9 expression and localization, as well as embryonic apoptosis. Hum Reprod 24:1373-1386

5. Bellemere G, Von Stetten O et al (2008) Retinoic acid increases aquaporin 3 expression in normal human skin. J Invest Dermatol 128:542-548

6. Bhalla K, Hwang BJ et al (2011) N-Acetylfarnesylcysteine is a novel class of peroxisome proliferator-activated receptor $\gamma$ ligand with partial and full agonist activity in vitro and in vivo. J Biol Chem 286:41626-41635

7. Bollag WB, Xie D et al (2007) A potential role for the phospholipase D2-aquaporin-3 signaling module in early keratinocyte differentiation: production of a phosphatidylglycerol signaling lipid. J Invest Dermatol 127:2823-2831

8. Boury-Jamot M, Sougrat M et al (2006) Expression and function of aquaporins in human skin: is aquaporin-3 just a glycerol transporter? Biochim Biophys Acta 1758:1034-1042

9. Cao C, Sun Y et al (2006) EGFR-mediated expression of aquaporin-3 is involved in human skin fibroblast migration. Biochem $\mathrm{J}$ 400:225-234

10. Chrispeels MJ, Agre P (1994) Aquaporins: water channel proteins of plant and animal cells. Trends Biochem Sci 19:421-425

11. Desrosiers RR, Gauthier F et al (2000) Modulation of Rho and cytoskeletal protein attachment to membranes by a prenylcysteine analog. J Biol Chem 275:14949-14957

12. Dietrich A, Scheer A et al (2003) Studies on G-protein alpha.betagamma heterotrimer formation reveal a putative S-prenylbinding site in the alpha subunit. Biochem J 376:449-456

13. Draelos Z (2012) Aquaporins: an introduction to a key factor in the mechanism of skin hydration. J Clin Aesthet Dermatol 5:53-56

14. Fernandez JR, Rouzard K et al (2012) SIG1273: a new cosmetic functional ingredient to reduce blemishes and Propionibacterium acnes in acne prone skin. J Cosmet Dermatol 11:272-278

15. Fernandez JR, Rouzard K et al (2015) Anti-inflammatory and anti-bacterial properties of tetramethylhexadecenyl succinyl cysteine (TSC): a skin-protecting cosmetic functional ingredient. Int J Cosmet Sci 37:129-133

16. Fernandez JR, Rouzard K et al (2016) In vitro and clinical evaluation of SIG1273: a cosmetic functional ingredient with a broad spectrum of anti-aging and antioxidant activities. J Cosmet Dermatol 15:150-157

17. FoggVC Azpiazu I et al (2001) Role of the gamma subunit prenyl moiety in $G$ protein beta gamma complex interaction with phospholipase Cbeta. J Biol Chem 276:41797-41802

18. Garcia N, Gondran C et al (2011) Impact of AQP3 inducer treatment on cultured human keratinocytes, ex vivo human skin and volunteers. Int J Cosmet Sci 33:432-442

19. Gordon JS, Wolanin PM et al (2008) Topical $N$-acetyl- $S$-farnesylL-cysteine inhibits mouse skin inflammation, and unlike dexamethasone, its effects are restricted to the application site. J Investig Dermatol 128:643-654

20. Gu LY, Qiu LW et al (2013) Expression of aquaporin 3 and aquaporin 9 is regulated by oleic acid through the PI3 K/Akt and p38 MAPK signaling pathways. Zhonghua Gan Zang Bing Za Zhi 21:753-758. doi:10.3760/cma.j.issn.1007-3418.2013.10.008

21. Hara M, Ma T, Verkman AS (2002) Selectively reduced glycerol in skin of aquaporin-3-deficient mice may account for impaired skin hydration, elasticity, and barrier recovery. J Biol Chem 277:46616-46621

22. Hara-Chikuma M, Verkman AS (2008) Roles of aquaporin-3 in the epidermis. J Invest Dermatol 128:2145-2151

23. Hara-Chikuma M, Satooka $\mathrm{H}$ et al (2015) Aquaporin-3-mediated hydrogen peroxide transport is required for NF- $\mathrm{KB}$ signalling in keratinocytes and development of psoriasis. Nat Commun 6:7454

24. Haratake A, Uchida Y et al (1997) Intrinsically aged epidermis displays diminished UVB-induced alterations in barrier function associated with decreased proliferation. J Invest Dermatol 108:319-323

25. Huzoor-Akbar Wang W et al (1993) Protein prenylcysteine analog inhibits agonist-receptor-mediated signal transduction in human platelets. Proc Natl Acad Sci USA 90:868-872

26. Jeon BK, Kang MK et al (2014) EPA attenuates ultraviolet radiation-induced downregulation of aquaporin-3 in human keratinocytes. Arch Pharm Res 38:1552-1560

27. Jiang YJ, Kim P et al (2011) PPARgamma activators stimulate aquaporin 3 expression in keratinocytes/epidermis. Exp Dermatol 20:595-599 
28. Jungersted JM, Bomholt $\mathrm{J}$ et al (2013) In vivo studies of aquaporin 3 and 10 in human stratum corneum. Arch Dermatol Res 305:699-704

29. Kim NH, Lee AY (2010) Reduced aquaporin 3 expression and survival of keratinocytes in the depigmented epidermis of vitiligo. J Invest Dermatol 130:2231-2239

30. Kloog Y, Cox AD (2004) Prenyl-binding domains: potential targets for Ras inhibitors and anti-cancer drugs. Semin Cancer Biol 14:253-261

31. Lee Y, Je YJ et al (2012) Changes in transepidermal water loss and skin hydration according to expression of aquaporin-3 in psoriasis. Ann Dermatol 24:168-174

32. Li Y, Liu Y et al (2010) UV irradiation induces Snail expression by AP-1 dependent mechanism in human skin keratinocytes. J Dermatol Sci 60:105-113

33. Marchini G, Stabi B et al (2003) Impact of AQP3 inducer treatment on cultured human keratinocytes, ex vivo human skin and volunteers. Pediatr Dermatol 20:377-384

34. Marshall CJ (1993) Protein prenylation: a mediator of proteinprotein interactions. Science 259:1865-1866

35. Melchionna R, Bellavia $G$ et al (2012) C/EBP $\gamma$ regulates wound repair and EGF receptor signaling. $J$ Invest Dermatol 132:1908-1917

36. Mor A, Aizman E et al (2013) Immunomodulatory properties of farnesoids: the new steroids? Curr Med Chem 20:1218-1224

37. Mor A, Haklai R et al (2011) Inhibition of contact sensitivity by farnesylthiosalicylic acid-amide, a potential Rap1 inhibitor. J Invest Dermatol 131:2040-2048

38. Olsson M, Broberg A et al (2006) Increased expression of aquaporin 3 in atopic eczema. Allergy 61:1132-1137

39. Papaharalambus C, Sajjad W et al (2005) Tumor necrosis factor alpha stimulation of Rac1 activity. Role of isoprenylcysteine carboxylmethyltransferase. J Biol Chem 280:18790-18796

40. Paz ML, Ferrari A (2008) Time-course evaluation and treatment of skin inflammatory immune response after ultraviolet B irradiation. Cytokine 44:70-77

41. Philips MR, Pillinger MH et al (1993) Carboxyl methylation of Ras-related proteins during signal transduction in neutrophils. Science 259:977-980

42. Rojek A, Praetorius J (2008) A current view of the mammalian aquaglyceroporins. Annu Rev Physiol 70:301-327
43. Scheer A, Gierschik P (1993) Farnesylcysteine analogues inhibit chemotactic peptide receptor-mediated G-protein activation in human HL-60 granulocyte membranes. FEBS Lett 319:110-114

44. Seleit I, Bakry OA et al (2015) Evaluation of aquaporin-3 role in nonmelanoma skin cancer: an immunohistochemical study. Ultrastruct Pathol 39:306-317

45. Seleit I, Bakry OA, El Rebey HS, El-Akabawy G, Hamza G (2017) Is aquaporin-3 a determinant factor of intrinsic and extrinsic aging? An immunohistochemical and morphometric study. Appl Immunohistochem Mol Morphol 25:49-57. doi:10. 1097/PAI.0000000000000265

46. Shan SJ, Xiao T et al (2012) Kanglaite attenuates UVB-induced down-regulation of aquaporin-3 in cultured human skin keratinocytes. Int J Mol Med 29:625-629

47. Sougrat R, Morand M et al (2002) Functional expression of AQP3 in human skin epidermis and reconstructed epidermis. J Invest Dermatol 118:678-685

48. Stimmel JB, Deschenes RJ et al (1990) Evidence for an $S$-farnesylcysteine methyl ester at the carboxyl terminus of the Saccharomyces cerevisiae RAS2 protein. Biochemistry 29:9651-9659

49. Sugiyama Y, Yamazaki K et al (2014) Analysis of aquaporin 9 expression in human epidermis and cultured keratinocytes. FEBS Open Bio 4:611-616

50. Volker C, Miller RA et al (1991) Effects of farnesylcysteine analogs on protein carboxyl methylation and signal transduction. J Biol Chem 266:21515-21522

51. Voss KE, Bollag RJ et al (2011) Abnormal aquaporin-3 protein expression in hyperproliferative skin disorders. Arch Dermatol Res 303:591-600

52. Winter-Vann AM, Casey PJ (2005) Post-prenylation-processing enzymes as new targets in oncogenesis. Nat Rev Cancer $5: 405-412$

53. Wu Z, Uchi H et al (2014) Resveratrol inhibition of human keratinocyte proliferation via SIRT1/ARNT/ERK dependent downregulation of aquaporin 3. J Dermatol Sci 75:16-23

54. Xie H, Liu F et al (2013) Protective role of AQP3 in UVAinduced NHSFs apoptosis via Bcl2 up-regulation. Arch Dermatol Res 305:397-406 